\title{
Multiobjective-Optimized Design of a New UWB Antenna for UWB Applications
}

\author{
C. Moreno de Jong van Coevorden, ${ }^{1}$ M. Fernández Pantoja, ${ }^{1}$ Salvador G. García, \\ A. Rubio Bretones, ${ }^{1}$ R. Gómez-Martín, ${ }^{1}$ and K. Palmer ${ }^{2}$ \\ ${ }^{1}$ Department of Electromagnetism, University of Granada, Avenida Fuentenueva sn, 18071 Granada, Spain \\ ${ }^{2}$ Department of Electric and Electronic Engineering, University of Stellenbosch, Matieland 7602, South Africa
}

Correspondence should be addressed to Salvador G. García; salva@ugr.es

Received 20 November 2012; Revised 6 March 2013; Accepted 7 March 2013

Academic Editor: Michael Yan Wah Chia

Copyright (C) 2013 C. Moreno de Jong van Coevorden et al. This is an open access article distributed under the Creative Commons Attribution License, which permits unrestricted use, distribution, and reproduction in any medium, provided the original work is properly cited.

\begin{abstract}
A multiobjective genetic algorithm has been applied to design a new printed, bow-tie antenna for ultrawideband applications, that is, ground penetrating radar, short range and high data rate communications, and so forth. The ultrawideband performance with respect to antenna impedance and gain is achieved by an optimized resistive loading profile and flare angle. A low-cost prototype is manufactured and numerical simulations are validated with measurements.
\end{abstract}

\section{Introduction}

The antenna performance in ground penetrating radar (GPR) systems is measured by its ability to transmit and receive short pulses (on the order of a few nanoseconds), whose duration is a tradeoff between range resolution and penetration depth [1]. The transmission without distortion of these pulses needs ultrawideband (UWB), nondispersive antenna systems. Such ultrawideband antennas must exhibit a linear phase characteristic over the whole operating frequency band (so-called transient antenna [2]), apart from constant magnitude of the input impedance, polarization, and gain.

When an antenna is fed by a transient pulse (impulse antenna), the initial acceleration of the charges produces radiation from the feed point, which is the sole source of radiation until the traveling pulse reaches any antenna discontinuity. There, the current pulse that propagates along the antenna structure, is partially reflected, constituting secondary radiating sources [3]. Therefore, the time-domain antenna response can be divided into two parts: the main pulse and the ringing region. The main pulse results from the direct radiation of the excitation pulse at the feed point, while radiation originating at antenna discontinuities, gives rise to the ringing region. Depending on the relation between width of the pulse and size of the antenna, these regions could overlap.

A design goal for impulse GPR antennas is the removal or minimization of the ringing region. The main method to achieve this is to establish a proper distribution of resistive loads along the antenna to diminish the reflections of the current pulses at antenna discontinuities [4-6]. As a result, the input-impedance bandwidth of the antenna is increased [7]. However, the enhancement of the ultrawideband characteristics of an antenna, by loading its structure, reduces the antenna gain due to ohmic losses. The design of such antennas is a multiobjective engineering problem with opposite goals, where a tradeoff between bandwidth and gain needs to be found.

One kind of antenna widely used in GPR applications is the solid bow-tie antennas $[4,8]$ being simple to design and having ultrawideband impedance properties. However, in some cases, the use of the wire bow-tie antenna or strip bow-tie antenna may be advantageous, as they are more easily loaded with resistors than their solid counterparts and have adaptive properties $[9,10]$. In [11], a microgenetic optimization algorithm (GA) was used to optimize the input impedance bandwidth of a thin-wire bow-tie antenna. The result was an antenna with a very high impedance bandwidth 
but a low gain. In an effort to increase the directivity of the antenna, a new thin-wire design was proposed in [12], where the front-to-back ratio and the broadband behavior of the input impedance were simultaneously optimized by means of a multiobjective GA. Improved directivity was achieved by bending the two arms of the antenna in a horn-like way, while the broadband impedance characteristics were accomplished by discrete resistive loading of the antenna wires. The result was greater broadside direction gain but at the expense of augmenting the antenna profile.

In this paper, a new optimized printed-strip bow-tie antenna is proposed. With the aim of using a multiobjective GA for the simultaneous optimization of three following antenna parameters: $S_{11}$ bandwidth, gain bandwidth, and gain in the broadside direction. This approach goes beyond simply seeking an antenna with high impedance bandwidth, as in the case described in [11]. Moreover, the possibility of using different load profiles on the different strips that compose the antenna geometry is allowed, so that it is possible to find better designs than in $[11,12]$, where the same load profile was used in all the antenna wires. The only condition imposed on the load profile is to have quadrangular symmetry. The optimization was carried out in two steps. First, a flat, thin-wire bow-tie antenna was designed by hybridizing an in-house multiobjective GA code with the method-of-moments code NEC [13] for modeling the thinwire antenna response. Subsequently, in order to facilitate the construction of a prototype, the wire antenna was converted into a printed strip using the same surface area rule of thumb [14]. Finally, the equivalent printed antenna prototype was built and its measured $S_{11}$ parameter and gain were found to be in good agreement with the numerical results predicted by the commercial code CST Microwave Studio. (This two-step designing procedure is followed because the computation speed of the hybrid GA-NEC code is much faster than the GA-CST.)

The paper is organized as follows. Section 2 describes the parameters that characterize the antenna to be optimized, the specific strategy followed to produce a new UWB optimized design and the numerical results corresponding to that design. In Section 3 , the $S_{11}$ parameter and antenna gain are measured and compared with numerical simulations. Conclusions are discussed in Section 4, where future directions of investigation are also outlined. Finally, in Appendix, a stepby-step description of the process followed to transform a thin-wire, bow-tie antenna to an equivalent printed antenna is given.

\section{Multiobjective Antenna Optimization}

There are many strategies to handle multiobjective optimization problems: Vector Evaluated Genetic Algorithm [15], linear aggregation of objectives [16], or the most widely used, which is based on Pareto dominance [17]. In this paper, we will use this last strategy due to its high rate of success in electrical and electronic engineering applications $[18,19]$. In particular, we chose the nondominated sorting genetic algorithm in the revised version of Deb et al. (NSGAII) because

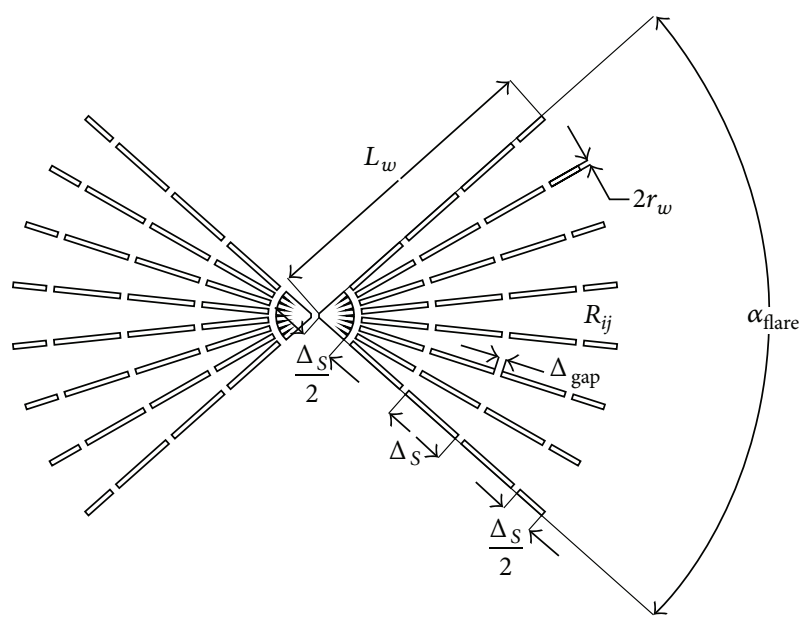

FIgURE 1: Geometry of the thin-wire bow-tie antenna to be optimized. $L_{w}=75 \mathrm{~mm}, \Delta_{S}=18.75 \mathrm{~mm}, \Delta_{\text {gap }}=2 \mathrm{~mm}$, and radius $r_{w}=0.65 \mathrm{~mm}$.

of its easy implementation, remarkable ability to reach the true Pareto front in mathematical test functions, and less computational complexity than other similar algorithms [17].

In this section, the system to be optimized is first described, setting what parameters can be tuned up and what parameters are kept fixed. Later, the basics of the optimization algorithm that is used in this work is outlined, ending with the presentation of the numerical results from the optimization process.

2.1. Description of the Optimized System. The geometry of the thin-wire bow-tie antenna proposed for optimization is shown in Figure 1. It is made up by $N_{d}$ dipoles $\left(2 * N_{d}\right.$ wires) forming a fanlike structure and fed at the center common point. Equally spaced gaps of width $\Delta_{\text {gap }}$ separated by a distance of $\Delta_{S}$ are inserted in the wires. At these gaps, chip resistors are soldered in order to enhance the ultrawideband characteristics of the antenna to be optimized in the frequency range between $0.5 \mathrm{GHz}$ and $3 \mathrm{GHz}$, which is a typical band in GPR applications. Four different resistors are located on each arm, but, since the maximum of the radiation pattern is required to be in the broadside direction, the distribution of resistors is forced to have quadrangular symmetry and therefore only $2 * N_{d} / 4$ different resistors will be considered in total for an antenna design. The number of dipoles were chosen to be $N_{d}=8$ because, although the UWB behavior of the antenna increases with the number of wires, no noticeable improvements are found over $N_{d}=8$ [20]; therefore, the load profile will consist in only 16 different resistors.

Among the parameters that define the antenna, the ones that will be allowed to vary during the optimization process are the exterior flare angle $\alpha_{\text {flare }}$, keeping the wires equiangular spaced, and the values of the resistors $R_{i j}$ at wire $i$, $i=1, \ldots, 4$ and position $j$ on that wire, $j=1, \ldots, 4$, where $i=$ 1 is the most external wire and $j=1$ the resistor placed in the vicinity of the feed point. The remaining parameters are kept 


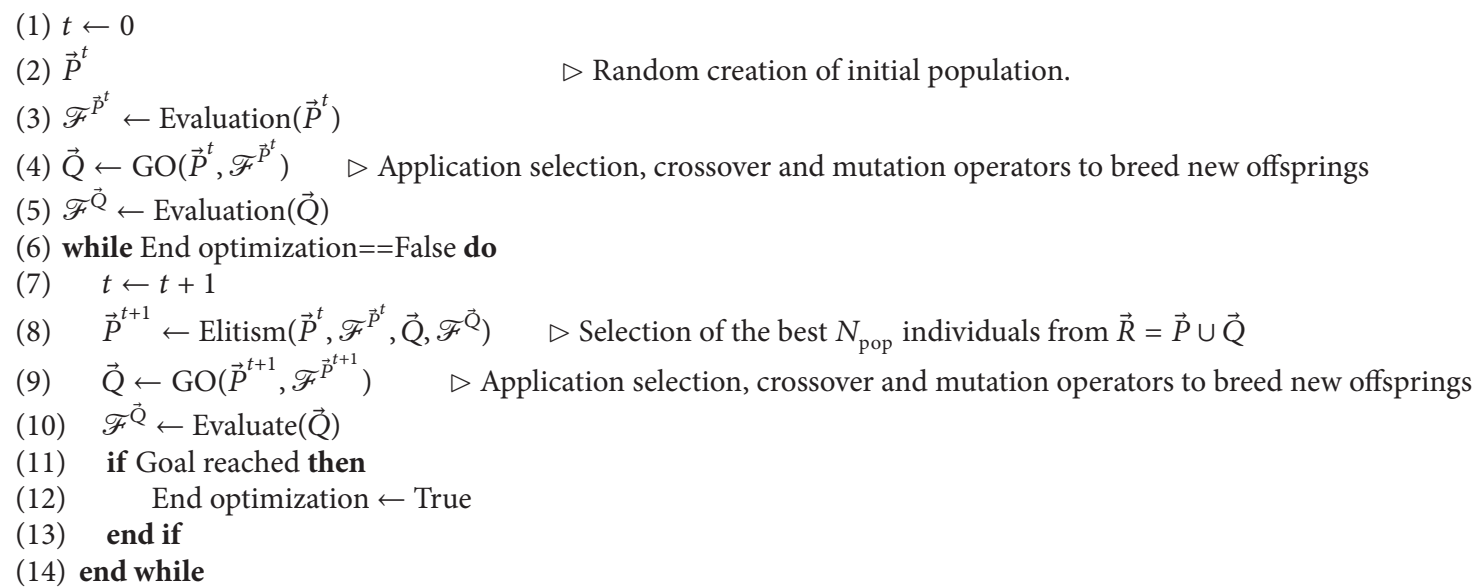

Algorithm 1: Description of the NSGAII.

fixed. The wires are chosen to have a radius of $r_{w}=0.65 \mathrm{~mm}$ while their length is $L_{w}=75 \mathrm{~mm}$ and the resistors, of body length $\Delta_{\text {gap }}=2 \mathrm{~mm}$ (0805 SMD chip resistors), are equally spaced over the wires separated by a distance $\Delta_{S}=18.75 \mathrm{~mm}$.

\subsection{Antenna Optimization Using a Multiobjective GA. GAs} are optimization algorithms based on the theories of evolution and genetics [21]. GAs are iterative algorithms that consider a population of individuals, each individual representing a potential solution of the problem at hand, which is described by a set of genes. The process starts by evaluating the quality of individuals in the initial population by calculating a representative function, named fitness function, which is defined by the designer in terms of the expected performance of the optimized antenna. The best designs are selected and undergo the genetic operations of crossover and mutation, resulting in a new generation of individuals to be evaluated again. Crossover is usually accomplished by randomly selecting two individuals (called parents) and generating another two (called offsprings) by mixing the genes carried by the parents in some specified fashion. Mutation changes a gene with a certain probability within the allowed values in the design. This process is continued until a stop criterion is met.

The specific optimization algorithm that is used in the present work is the NSGAII. This algorithm is used to optimize, simultaneously, the $S_{11}$ parameter and gain $(G)$ of the model proposed above, seeking to achieve the widest possible bandwidth in both magnitudes, with the highest value of the latter. As fitness functions to evaluate the performance of each individual, the following were chosen.

$f_{S_{11}}$ : the width of the band where $S_{11}$ is below $-10 \mathrm{~dB}$, calculated using as characteristic impedance, $Z_{0}=$ $\operatorname{re}\left(\bar{Z}_{\text {in }}\right)$, being $\bar{Z}_{\text {in }}$ the mean value of the real part of $Z_{\text {in }}$ in the frequency band of interest. With this, the algorithm searches antennas having input impedances with real parts near to $\operatorname{re}\left(\bar{Z}_{\text {in }}\right)$ and imaginary parts as close to 0 as possible.
$f_{G}$ : the width of the band where the gain is between its maximum value and $3 \mathrm{~dB}$ below it.

$f_{G_{\max }}$ : The maximum value of the Gain in the frequency band of operation.

The NSGAII starts with the creation of a population $\left(\vec{P}^{0}\right)$ (the exponent $t$ of $\vec{P}^{t}$ means the iteration step of the optimization process) of $N_{\text {pop }}$ individuals (antenna designs) with its parameters randomly chosen from a uniform distribution. In the present case, each antenna is represented by a real number, coding the flare angle $\alpha_{\text {flare}}$, and an integer matrix of 16 rows of three integers $X_{1}, X_{2}$ which defines the value of each of the 16 different resistors of the structure as $R=X_{1} \times 10^{X_{2}} \Omega$. After a preliminary study, the search space was reduced to the one defined by the discrete values $X_{1} \in[0,99]$ and $X_{2} \in[0,1]$ and the continuous interval $\alpha_{\text {flare }} \in\left[60^{\circ}, 120^{\circ}\right]$, thereby speeding up the convergence of the optimization process. The main cycle of the iterative process (see Algorithm 1) starts with the evaluation of each individual of the population by computing $Z_{\text {in }}$ and $G$ over the frequency band of interest by means of the method-of-moments-based frequency-domain code NEC. Then, the three fitness functions defined above are calculated for each individual and stored in a matrix $\mathscr{F}^{\vec{P}^{t}}$. After the entire population is evaluated and the most promising solutions are selected, genetic operators (GO) are applied to the population to generate the new generation of potential solutions or offspring $(\vec{Q})$ from the previous generation $\left(\vec{P}^{t}\right)$.

The specific genetic operators we employed were as follows. First, a binary tournament selection was used to identify the best individuals within the population. This operator randomly picks two individuals from the previous population and chooses the superior solution for a future crossover following the Pareto domination rules. As a mechanism to recombine the features of two individuals previously selected, a hybrid real-discrete crossover operator is chosen, where the angle $\alpha_{\text {flare }}$ is recombined via simulated binary crossover 
(1) for $j \leftarrow 1,3$ do

(2) $\quad u \leftarrow U[0,1]$

(3) if $u<p_{\text {res }}(j)$ then $\quad \triangleright$ Decision of muting one of the values that define a resistors.

(4) $\quad X_{j} \in U\left[0, \kappa_{j}\right]$

(5) end if

(6) end for

Algorithm 2: Discrete mutation of genes corresponding to resistors.

TABLE 1: Load profile of the selected antenna design. The $i$ values run in columns, the $j$ in rows.

\begin{tabular}{lcccc}
\hline$R_{i j}$ & 1 & 2 & 3 & 4 \\
\hline 1 & 24 & 0 & 11 & 39 \\
2 & 270 & 20 & 0 & 12 \\
3 & 790 & 33 & 14 & 96 \\
4 & 59 & 170 & 5 & 230 \\
\hline
\end{tabular}

[22], and the resistors by means of a two point crossover [21]. The polynomial distribution introduced by Deb [23] is applied to mutate $\alpha_{\text {flare }}$ while to mutate the resistors the algorithm shown in Algorithm 2 is used. Among these operators, the specific multiobjective genetic operator is the tournament selection, in which the dominance in the pareto sense is implemented.

The values of parameter $\kappa$ in Algorithm 2 define the variation range of each variable $X_{j}$, where $\kappa_{j}=(9,9,2)$. On the other hand, after a short parametric study, it was found that the probability rate $p_{\text {res }}=(0.2,1,0.05)$ gives satisfactory results. This probability was chosen so that, after the individual was selected to mutate (according to the muting rate $p_{m}$ ), the value mutated resistor was in the neighborhood of the previous one.

Finally, to create the next generation $\left(\vec{P}^{t+1}\right)$, an elitism operator was applied where the best $N_{\text {pop }}$ individuals from $\vec{P}^{t} \cup \vec{Q}^{t}$ were selected. This process was repeated until a proper set of solutions was found. For further details on the NSGAII algorithm, see [17].

2.3. Optimization Results. To ensure the convergence of the Pareto front, five independent runs of the optimization algorithm were executed with a population of $N_{\text {pop }}=100$ and crossover and mutation rates $p_{c}=0.8$ and $p_{m}=0.04$, respectively. The achieved nondominated set of solutions appears in Figure 2, which displays designs with $S_{11}$ bandwidths from $1.6: 1$ to $5.7: 1$, gain bandwidths from $1.0: 1$ to $5.4: 1$, and maximum gains from $-1.5 \mathrm{~dB}$ to $5.9 \mathrm{~dB}$. From this set of solutions, the designer can choose the best antenna that matches a particular application. In this paper, a compromise design (marked in red in Figure 2) is chosen to construct a prototype and measure its electrical properties.

The parameters of the chosen antenna are $\alpha_{\text {flare }}=84^{\circ}$ while the load profile is shown in Table 1.

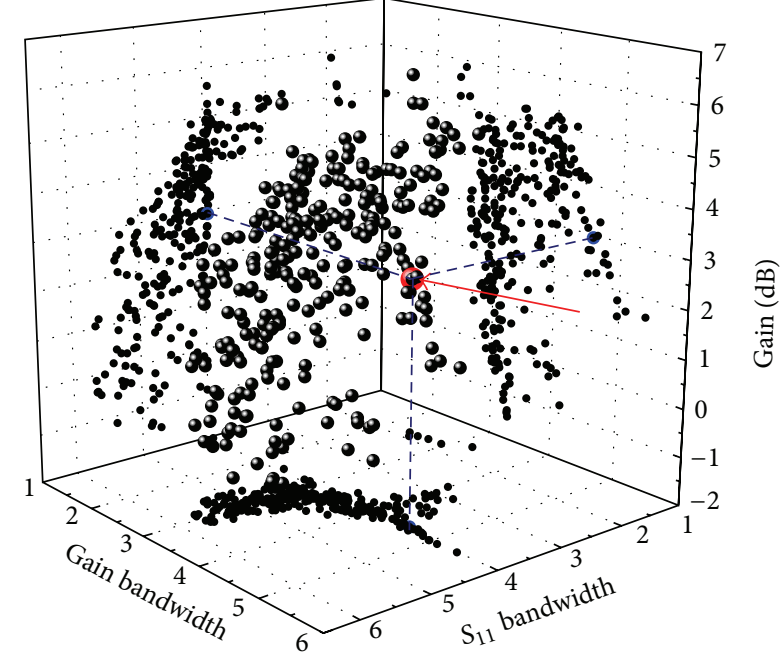

FIgURE 2: Pareto front and its projections at the end of the optimization process. The Pareto front is represented by black spheres while the selected design $\left(f_{S_{11}}=3.94, f_{G}=4.58, f_{G_{\max }}=2.93\right)$ is marked in red and pointed with an arrow.

\section{Experimental Validation of the Antenna Performance}

To illustrate the performance of the antenna design proposed in the previous section, two prototypes were fabricated and tested. One monopole over a ground plate fed with a coaxial connector and a dipole antenna fed by a $\lambda / 4$ balun. Both were made of metallic strips printed on FR-4 substrates $\left(\epsilon_{r}=4.9, \tan \delta=0.025\right)$ of thickness $\left.h=0.8 \mathrm{~mm}\right)$. A printed antenna model was chosen to facilitate its fabrication and testing in our laboratory facilities. Before the prototypes were built, numerical simulations were carried out by CST software, to determine how the antenna performance varied from the thin-wire model simulated by NEC and optimized by GA, to the printed strip model dealt with CST. The numerical results of all these experiments are presented in Appendix.

First, the monopole antenna shown in Figure 3 was built to measure its $S_{11}$ parameter. The antenna was printed on a FR-4 panel of dimension $132 \mathrm{~mm} \times 100 \mathrm{~mm}$, situated perpendicularly to an aluminum ground plane and fed with a $50 \Omega$ coaxial cable with its inner conductor attached to the antenna structure and its outer one to the ground plane. In order to know the minimum size of the ground 


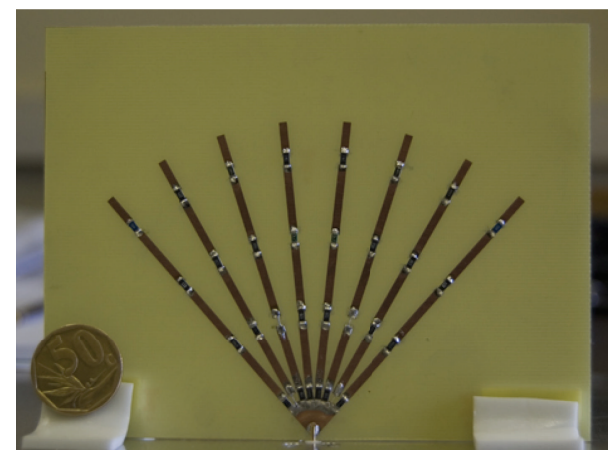

FIgURE 3: Monopole antenna for $S_{11}$ measurements.

plane, some numerical simulations were carried out in CST. The simulated antenna system was modeled as realistic as possible, including the FR-4 substrate, finite thickness ground plane and the coaxial connector feeding the antenna. These numerical simulations showed that, for $S_{11}$ calculations, a size of $297 \mathrm{~mm} \times 400 \mathrm{~mm}$ for the ground plane accurately approximates an infinite ground plane. The $S_{11}$ parameter was measured, in the frequency range from $500 \mathrm{MHz}$ to $3 \mathrm{GHz}$, by placing the antenna under test inside an anechoic chamber, and using an HP $8510 \mathrm{C}$ network analyzer. Figure 4 shows the measured return loss compared to the CST numerical results (the measured values were normalized to the mean value of the simulated antenna input resistance, in the frequency band considered, which was $Z_{0}=81 \Omega$ ). It can be seen that measurements and simulations match closely throughout the frequency band. Moreover, although it is not shown here, because the optimization was performed only up to $3 \mathrm{GHZ}$, it should be mentioned that the numerical return loss was below $-10 \mathrm{db}$ up to $8 \mathrm{GHz}$ rendering a numerical bandwidth in $S_{11}$ greater than $10: 1$.

Next, we proceeded to measure the antenna gain pattern but, unlike what happened with the $S_{11}$ parameter, it was found, the CST simulations indicated that the size of the ground plane needed by the gain pattern of the monopole antenna to match that of the dipole antenna was on the order of several meters. Since this was an impractical size for measurements in the anechoic chamber available at the University of Stellenbosch, as a second prototype, the whole dipole antenna was designed and fabricated. It was printed on a FR- 4 panel of dimension $(200 \times 205 \mathrm{~mm})$ and fed with a $\lambda / 4$ balun designed for $1.38 \mathrm{GHz}$ (see Figure 5). Measurements were carried out just at the frequency of $1.38 \mathrm{GHz}$, while numerical simulations were performed over the entire frequency range. Figure 6 plots, with a symbol line, the numerically calculated gain in the broadside direction, versus frequency, showing a $3 \mathrm{~dB}$ gain bandwidth of $1: 4.5$. A comparison between the computed and measured gain patterns at $1.38 \mathrm{GHz}$ is included in the inset of Figure 6, where a close matching between both results is noticed. The measured $S_{11}$ at $1.38 \mathrm{GHz}$ using the $\lambda / 4$ balun was $-8 \mathrm{~dB}$.

Finally, since a good time-domain performance is a primary requirement when designing an UWB antenna for

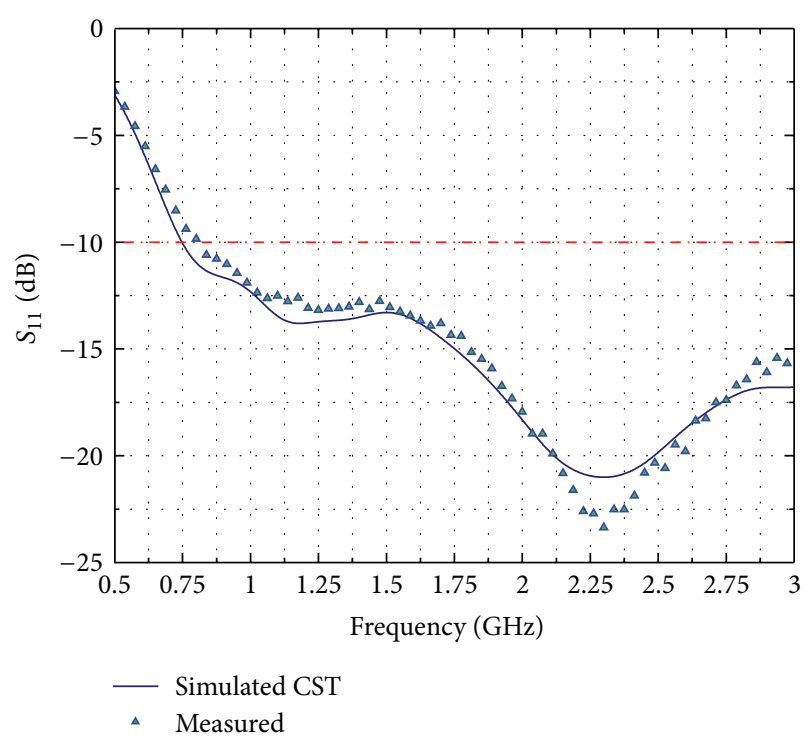

FIGURE 4: Simulated and measured $S_{11}$ parameter for the monopole strip bow-tie antenna.

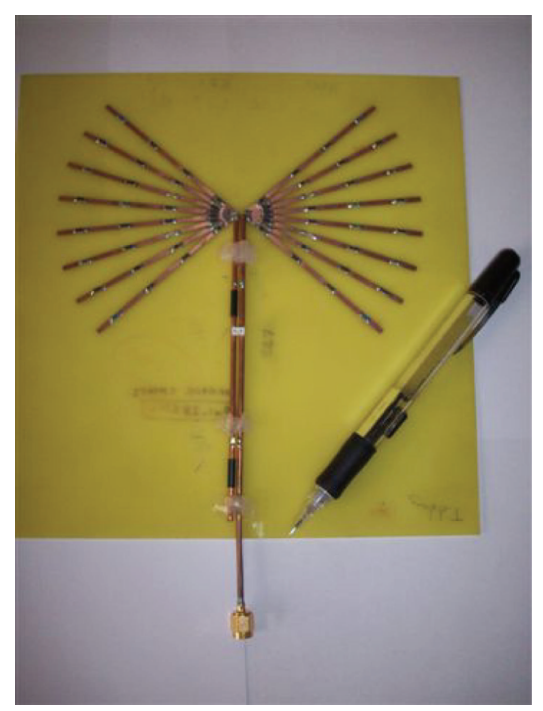

FIGURE 5: Strip, dipole, bow-tie antenna built for gain measurement. It is excited with a $\lambda / 4$ balun designed for $1.38 \mathrm{GHz}$.

GPR applications, the time-domain behavior of the stripdipole antenna has been investigated. The dipole antenna was excited at its center by a transient, voltage pulse with significant spectral components between $0.75 \mathrm{GHz}$ and $3 \mathrm{GHz}$ and its response was numerically calculated using CST. The shape of the voltage input signal, shown in a solid line in Figure 7, closely resembles that usually found in commercial GPR systems. It also has to be noted how the resistance loading has eliminated the multiple resonances associated to this type of antennas when unloaded [20]. These resonances are related to the presence of eigen modes which, in the case of loaded antennas, are shifted to lower frequencies, out of the frequency band of interest. 


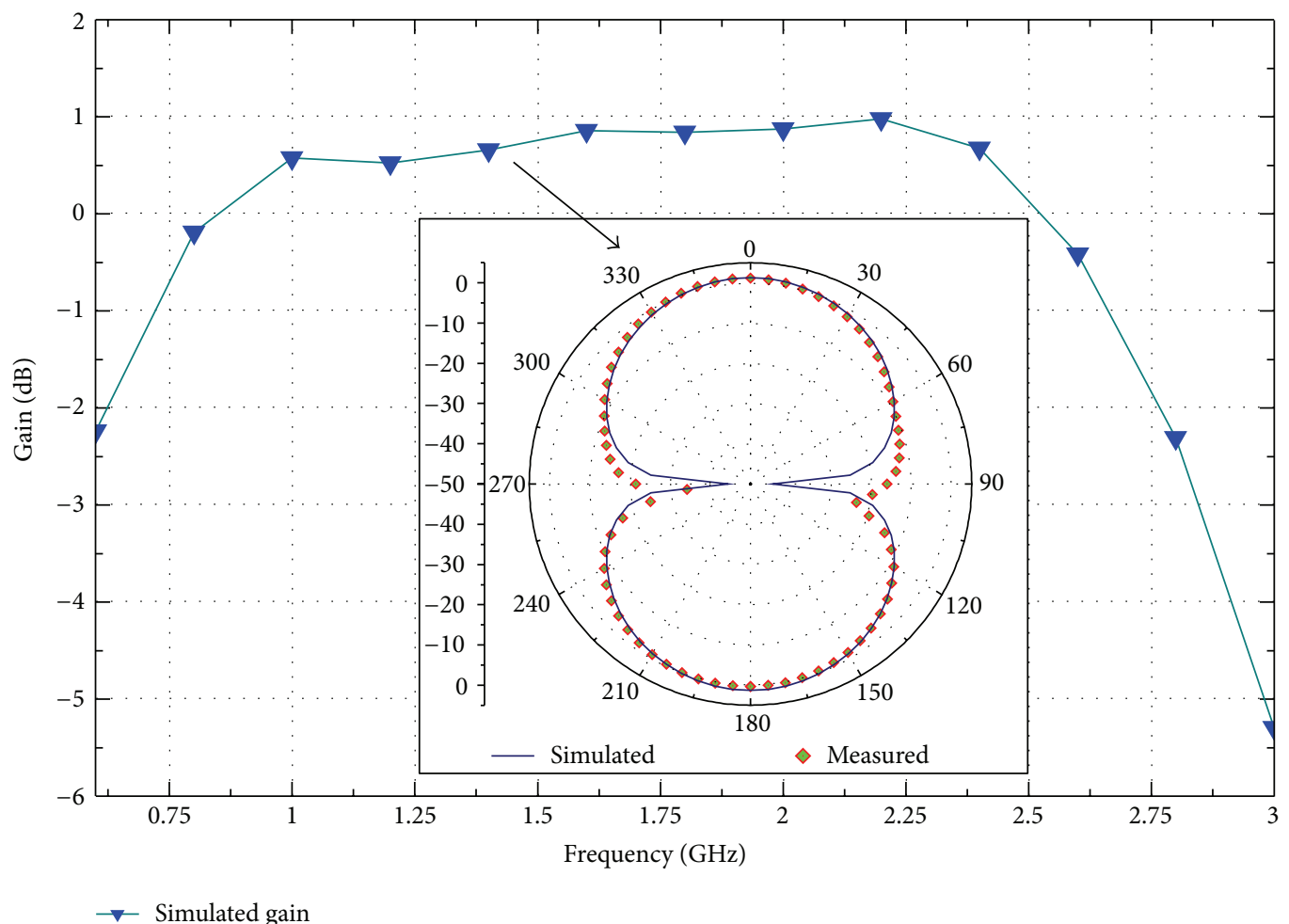

FIGURE 6: Simulated gain at the broadside direction over the frequency band of interest. Inset: simulated and measured $E$-plane, gain patterns of the dipole antenna at $1.38 \mathrm{GHz}$.

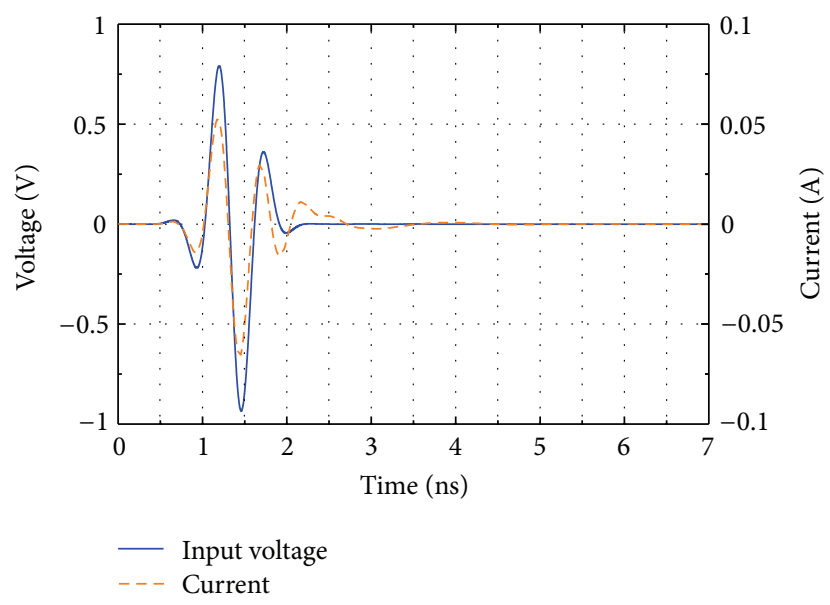

FIGURE 7: Time-domain response of the chosen antenna design: input voltage (solid line) and output current at the feed point (dashed line) versus time.

To quantify the level of late-time ringing in the antenna time-domain response, the fidelity between the input voltage and the output current at the feed point is calculated as the cross-correlation between both magnitudes using the expression [24]:

$$
\text { fidelity }_{12}=\left|\frac{\rho_{12}(t)}{\sqrt{\rho_{1}(0) \rho_{2}(0)}}\right|_{\max },
$$

where $\rho_{12}(t), \rho_{1}(0)$, and $\rho_{2}(0)$ are the cross-covariance and autocovariances of signals one and two, respectively. The fidelity compares the pulse shapes disregarding theirs amplitudes and time-delay factors. The value of the fidelity ranges from 0 (totally different signals) to 1 (the signals match perfectly). For the proposed antenna design, the fidelity factor computed for the input voltage and the current at the feed was found to be 0.937 . The current pulse at the feed point is plot versus time in Figure 7, and it can be seen that its shape is quite similar to that of the voltage input pulse with a very low late-time ringing.

It is also of great interest to investigate the ability of an UWB antenna to preserve the waveform of the radiated fields in different directions. In the time-domain, and for transmission mode, the quality of the radiated signal waveform in relation to the input voltage applied to the antenna terminals is measured by the fidelity between the time integral of the transmitted field and the input voltage [24]. The time-domain electric fields radiated in different directions in the $E$ and $H$ planes were calculated using CST. In all cases the fields were computed at a distance of $0.5 \mathrm{~m}$ from the antenna feed point. They are plotted in Figures 8 and 9. The simulated fidelity factors between the input voltage and the time-integrated radiated fields are given in Table 2 for different observation angles. It can be seen that the transmitted electric field in the $H$-plane presents high fidelity and its amplitude is almost constant for the different observation angles. Regarding the behavior of the electric field in the E-plane, Figure 9 shows how, on the one hand, the amplitude of the field decays 


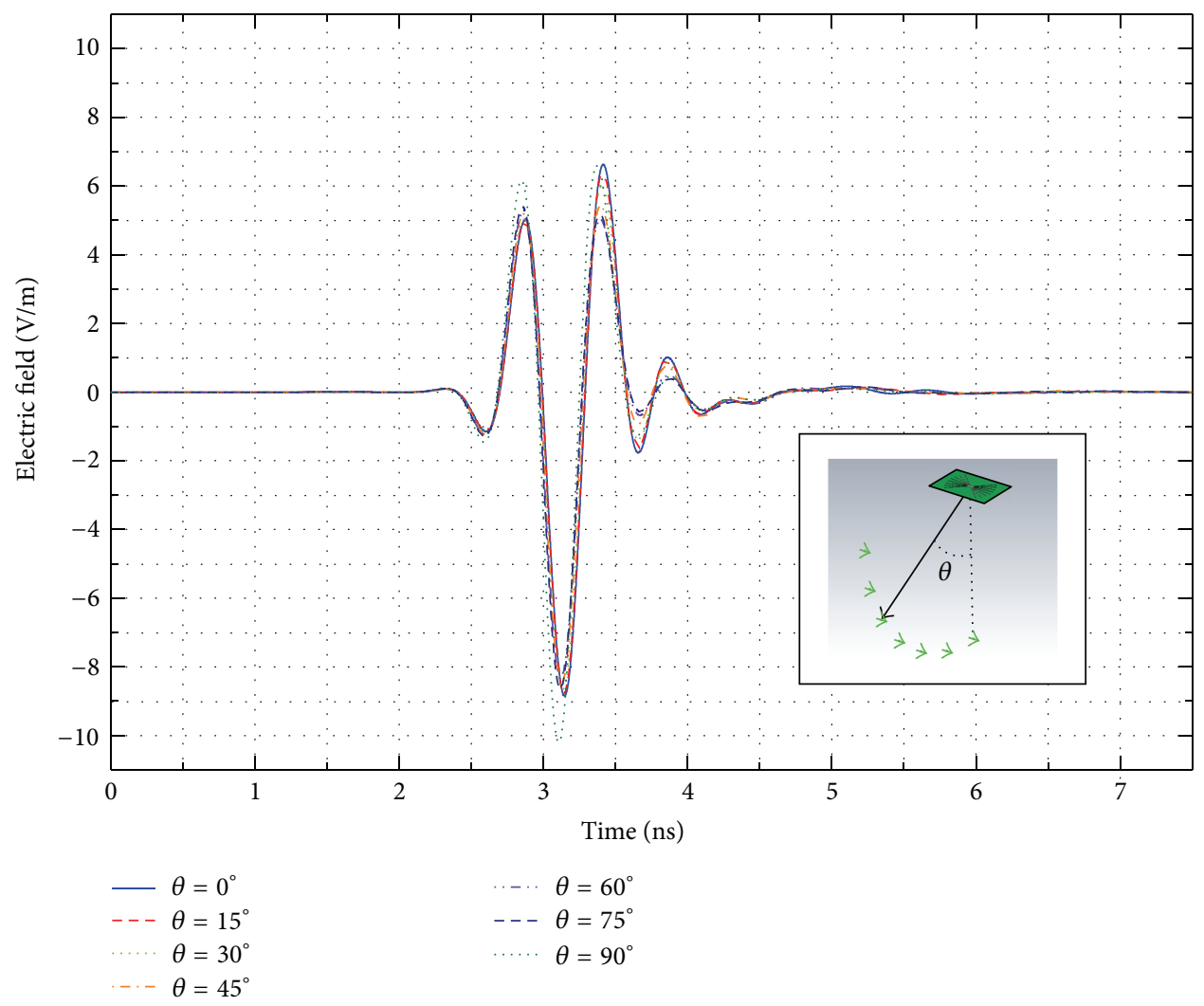

FIGURE 8: Simulated electric field for different azimuth angles in the $H$-plane calculated at a distance of $0.5 \mathrm{~m}$ from the antenna feed port.

TABLE 2: Fidelity of the radiated field at several observation angles.

\begin{tabular}{lcc}
\hline$\theta$ & $E$-plane & $H$-plane \\
\hline $0^{\circ}$ & 0.947 & 0.947 \\
$15^{\circ}$ & 0.943 & 0.945 \\
$30^{\circ}$ & 0.939 & 0.943 \\
$45^{\circ}$ & 0.930 & 0.933 \\
$60^{\circ}$ & 0.912 & 0.925 \\
$75^{\circ}$ & 0.774 & 0.923 \\
$90^{\circ}$ & 0.626 & 0.956 \\
\hline
\end{tabular}

as the observation angle is increased and, on the other hand, the field has high fidelity for observation angles $\theta \leq 60$ but it degrades dramatically for greater observation angles. These results are in accordance with the typical shape of dipoleantenna radiation patterns in the frequency domain, where the amplitude is almost constant in the $H$-plane, while in the $E$-plane varies from maximum values at broadside to minimum in the endfire direction [25].

\section{Conclusion}

A multiobjective GA has been employed to the design of novel UWB, thin-wire bow-tie antenna for GPR applications. The maximum antenna gain and the impedance and gain bandwidths are simultaneously optimized. The advantage of using a multiobjective GA is that a vast set of design solutions is found in an optimization run instead of only one, allowing the engineer to pick the one most suitable to a particular application. An specific design has been chosen on the Pareto-optimal front and, following the same surface rule, an equivalent printed-strip model of the selected thinwire antenna has been built and measured. The selected UWB antenna has been characterized in the frequency and time domains and good agreement was observed between numerical predictions and experimental results.

\section{Appendix}

\section{Strip Model of the Thin-Wire, Bow-Tie Antenna}

A printed antenna based on a thin-wire design requires the width of the printed strips to be chosen so that both models behave in the same manner. This is often done by using the same surface area rule [14]. In this appendix, we perform numerical simulations with CST to check whether this rule holds for this particular case.

In the thin-wire model, the area of each wire is $A_{w}=$ $2 L_{w} \pi r_{w}$, whereas in the printed antenna-model the area of each strip is $A_{s}=2 L_{w} w_{s}$, where $w_{s}$ is the strip width (the strip thickness is neglected). For the antenna design of radius of $0.65 \mathrm{~mm}$ proposed in this paper (see Figure 10), 


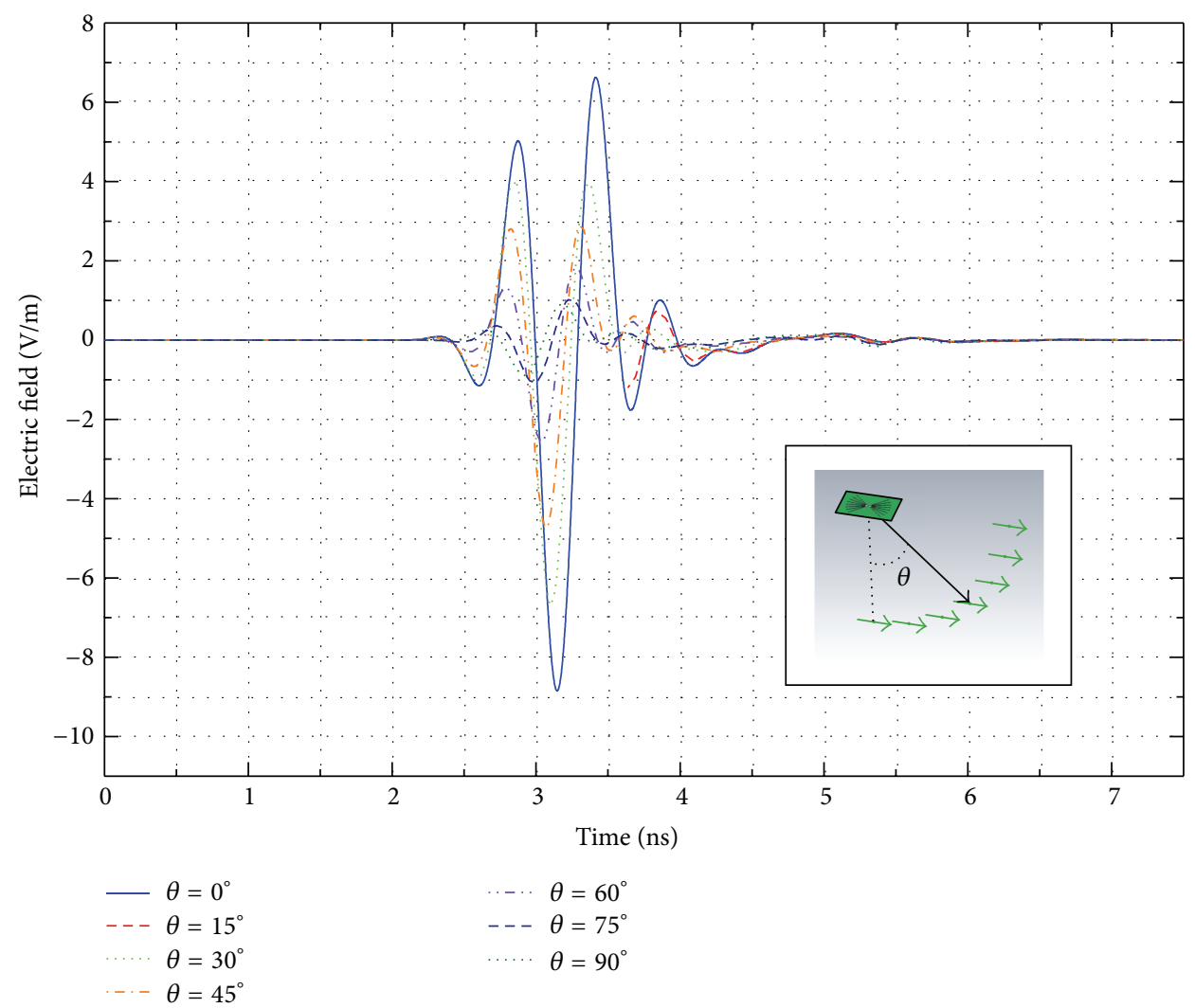

FIGURE 9: Simulated electric field for different azimuth angles in the E-plane calculated at a distance of $0.5 \mathrm{~m}$ from the antenna feed port.

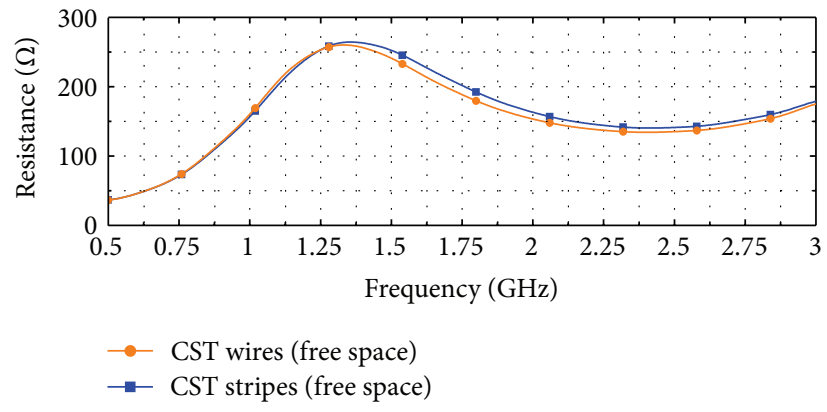

(a)

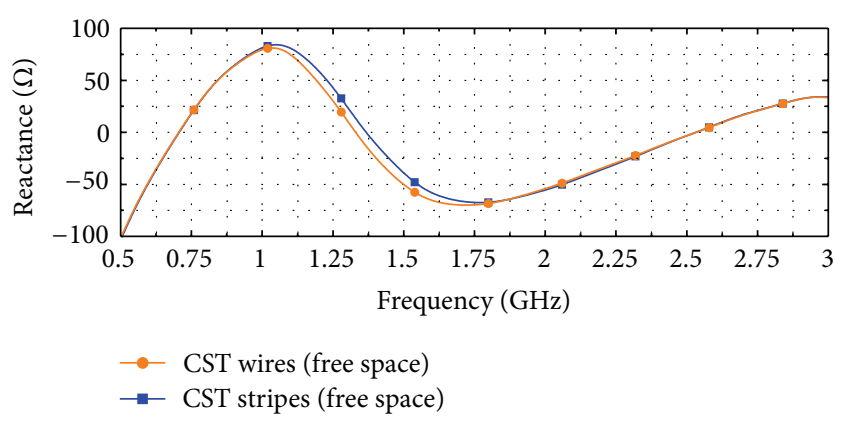

(b)

FIGURE 10: Simulated input impedance of the dipole thin-wire antenna proposed in this paper and a strip antenna that fulfills the same surface area. Both antennas are located in free space.

the same surface area rule yields $2 \mathrm{~mm}$-wide strips for the printed model. Figure 10 shows the good agreement between the input impedance of the $2 \mathrm{~mm}$-wide, strip, dipole antenna and that of its $0.65 \mathrm{~mm}$-radius, thin-wire counterpart, in the considered frequency range. Both antennas were located in free space.

\section{Acknowledgments}

The work described in this paper and the research leading to these results has received funding from the Spain-South African joint research project HS2006-0012, the European
Community's Seventh Framework Programme FP7/20072013 under Grant Agreement no. 205294 (HIRF SE project), from the Spanish National Projects TEC2010-20841-C0404, CSD2008-00068, PHB2009-0067-PC, from the Junta de Andalucia Project P09-TIC-5327, and from the Granada Excellence Network of Innovation Laboratories (GENIL) project.

\section{References}

[1] D. J. Daniels, Surface Penetrating Radar, IEE Radar, Navigation and Avionics Series, Institute of Electrical \& Electronics Enginee, 1996. 
[2] P. R. Foster, "Performance of ultrawideband antennas," in Ultrawideband Radar, vol. 1631 of Proceedings of SPIE, pp. 134145, January 1992.

[3] R. Gómez-Martín, A. R. Bretones, and S. G. García, "Some thoughts about transient radiation by straight thin wires," IEEE Antennas and Propagation Magazine, vol. 41, no. 3, pp. 24-33, 1999.

[4] K. L. Shlager, G. S. Smith, and J. G. Maloney, "Optimization of bow-tie antennas for pulse radiation," IEEE Transactions on Antennas and Propagation, vol. 42, no. 7, pp. 975-982, 1994.

[5] T. Wu and R. King, "The cylindrical antenna with nonreflecting resistive loading," IEEE Transactions on Antennas and Propagation, vol. 13, no. 3, pp. 369-373, 1988.

[6] M. Fernández-Pantoja, A. Monorchio, A. R. Bretones, and R. Gómez-Martín, "Direct GA-based optimisation of resistively loaded wire antennas in the time domain," Electronics Letters, vol. 36, no. 24, pp. 1988-1990, 2000.

[7] S. Lee and K. Mei, "Analysis of zigzag antennas," IEEE Transactions on Antennas and Propagation, vol. 18, no. 6, pp. 760-764, 1970.

[8] Y. Nishioka, O. Maeshima, T. Uno, and S. Adachi, "FDTD analysis of resistor-loaded bow-tie antennas covered with ferrite-coated conducting cavity for subsurface radar," IEEE Transactions on Antennas and Propagation, vol. 47, no. 6, pp. 970-977, 1999.

[9] A. A. Lestari, A. G. Yarovoy, and L. P. Ligthart, "Numerical and experimental analysis of circular-end wire bow-tie antennas over a lossy ground," IEEE Transactions on Antennas and Propagation, vol. 52, no. 1, pp. 26-35, 2004.

[10] A. A. Lestari, A. G. Yarovoy, and L. P. Ligthart, "Adaptive wire bow-tie antenna for GPR applications," IEEE Transactions on Antennas and Propagation, vol. 53, no. 5, pp. 1745-1754, 2005.

[11] C. M. de Jong van Coevorden, A. R. Bretones, M. FernándezPantoja, F. J. García Ruiz, S. G. García, and R. Gómez-Martín, "GA design of a thin-wire bow-tie antenna for GPR applications," IEEE Transactions on Geoscience and Remote Sensing, vol. 44, no. 4, pp. 1004-1009, 2006.

[12] C. Moreno de Jong van Coevorden, A. R. Bretones, M. Fernández-Pantoja et al., "Thin-wire antenna design for GPR applications using a multi-objective GA," Near Surface Geophysics, vol. 5, no. 1, pp. 23-28, 2007.

[13] G. Burke, A. Poggio, J. Logan, and J. Rockway, "NECnumerical electromagnetics code for antennas and scattering," in Antennas and Propagation Society International Symposium, vol. 17, pp. 147-150, 1979.

[14] A. C. Ludwig, "Wire grid modeling of surfaces," IEEE Transactions on Antennas and Propagation, vol. 35, no. 9, pp. 1045-1048, 1987.

[15] J. D. Schaffer, "Multiple objective optimization with vector evaluated genetic algorithms," in Proceedings of the 1st International Conference on Genetic Algorithms, pp. 93-100, 1985.

[16] P. Hajela and C. Y. Lin, "Genetic search strategies in multicriterion optimal design," Structural Optimization, vol. 4, no. 2, pp. 99-107, 1992.

[17] K. Deb, A. Pratap, S. Agarwal, and T. Meyarivan, "A fast and elitist multiobjective genetic algorithm: NSGA-II," IEEE Transactions on Evolutionary Computation, vol. 6, no. 2, pp. 182197, 2002.

[18] S. Cui, A. Mohan, and D. S. Weile, "Pareto optimal design of absorbers using a parallel elitist nondominated sorting genetic algorithm and the finite element-boundary integral method,"
IEEE Transactions on Antennas and Propagation, vol. 53, no. 6, pp. 2099-2107, 2005.

[19] Y. Kuwahara, "Multiobjective optimization design of Yagi-Uda antenna," IEEE Transactions on Antennas and Propagation, vol. 53, no. 6, pp. 1984-1992, 2005.

[20] C. M. de Jong van Coevorden, Optimized antenna design using genetic algorithms [Ph.D. thesis], Electromagnetismo, Universidad de Granada, 2008.

[21] K. A. de Jong, Analysis of the behavior a class of genetic adaptative systems [Ph.D. thesis], Dept. Comput. Commun. Sci., Univ. Michigan, Ann Arbor, Mitch, USA, 1975.

[22] K. Deb and R. B. Agrawal, "Simulated binary crossover for continuous search space," Complex Systems, vol. 9, pp. 115-148, 1995.

[23] K. Deb, "An efficient constraint handling method for genetic algorithms," Computer Methods in Applied Mechanics and Engineering, vol. 186, no. 2-4, pp. 311-338, 2000.

[24] O. E. Allen, D. A. Hill, and A. R. Ondrejka, "Time-domain antenna characterizations," IEEE Transactions on Electromagnetic Compatibility, vol. 35, no. 3, pp. 339-345, 1993.

[25] C. A. Balanis, Antenna Theory, Analisys and Design, John Wiley \& Sons, New York, NY, USA, 1982. 

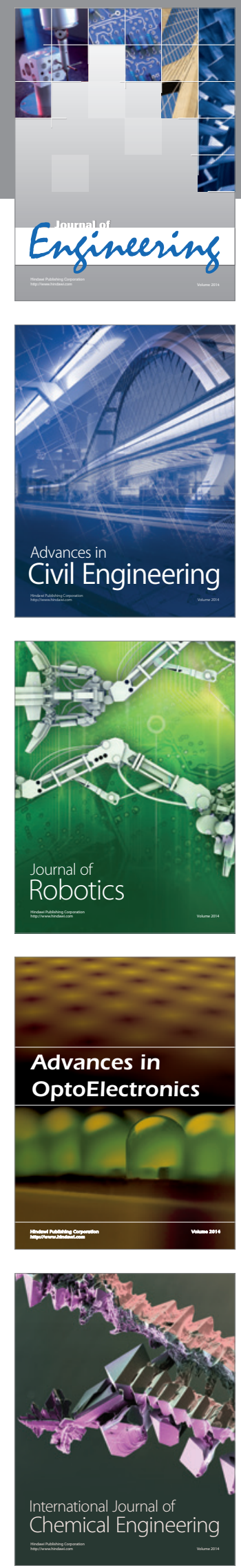

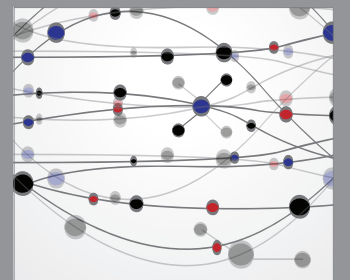

The Scientific World Journal
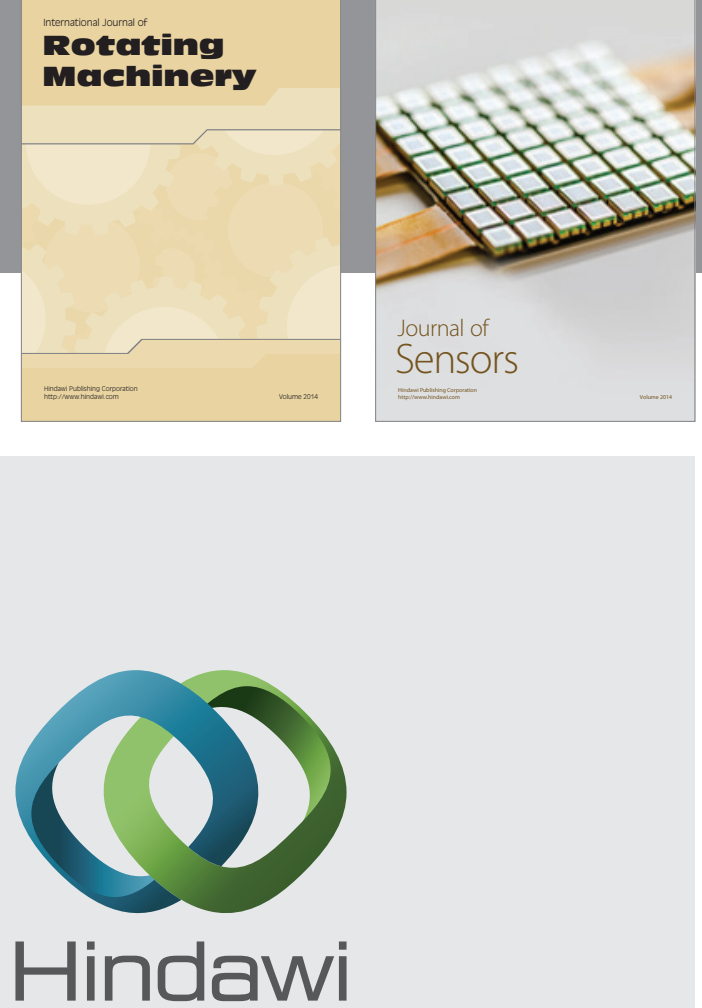

Submit your manuscripts at http://www.hindawi.com
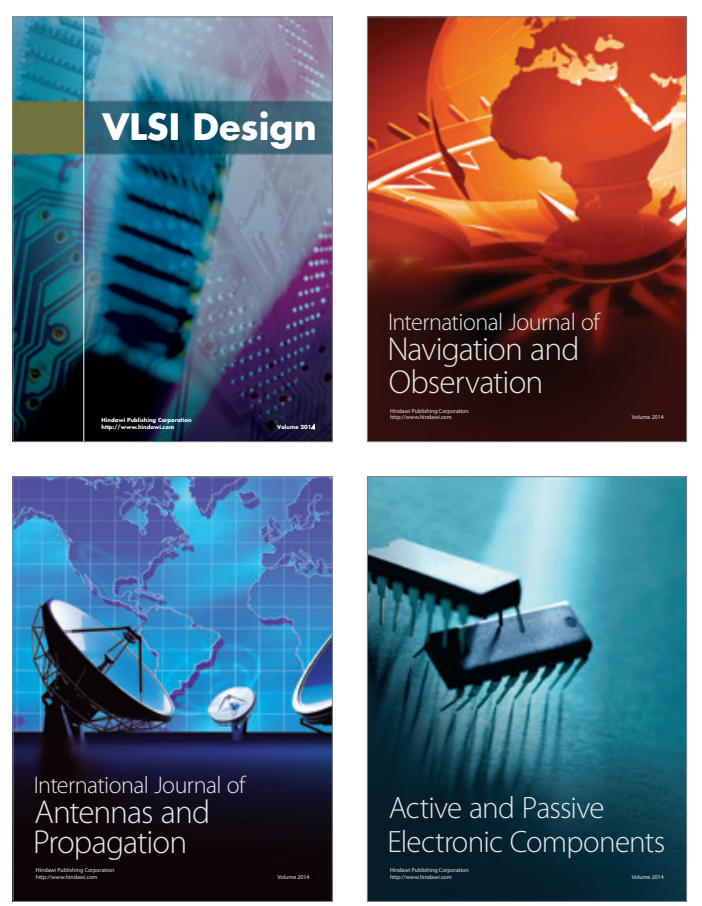
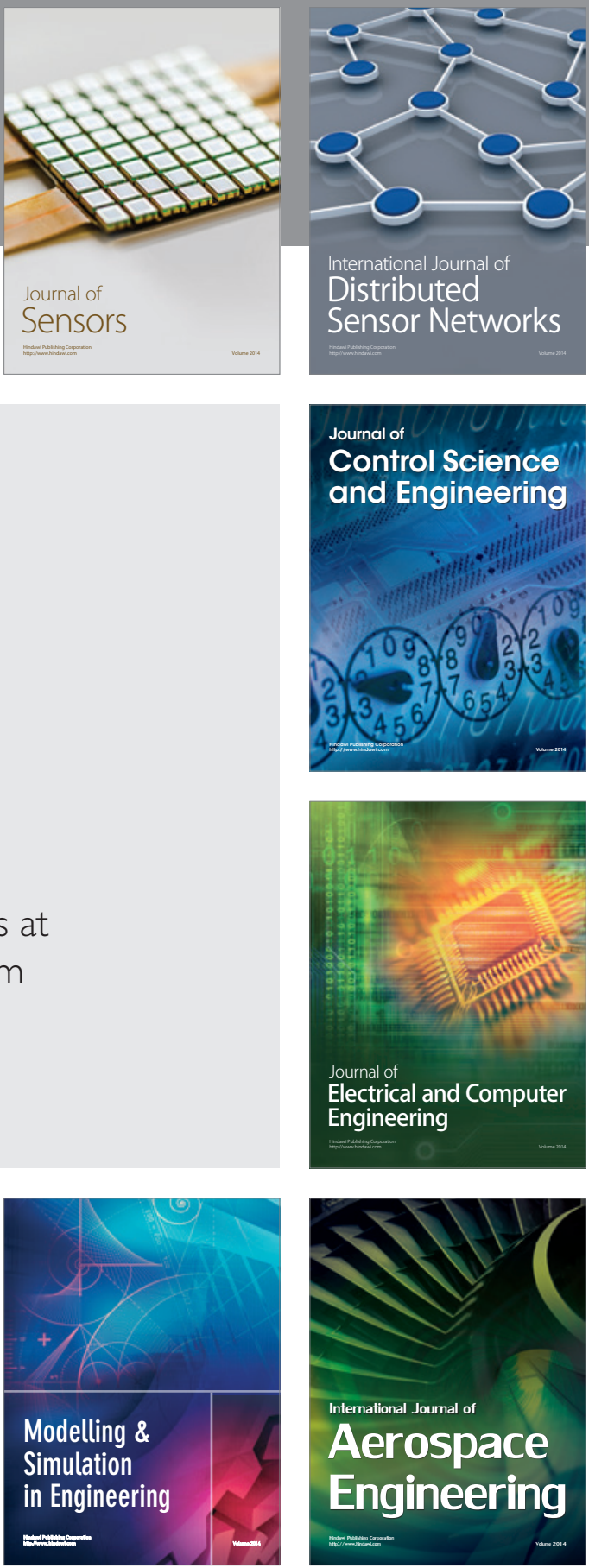

Journal of

Control Science

and Engineering
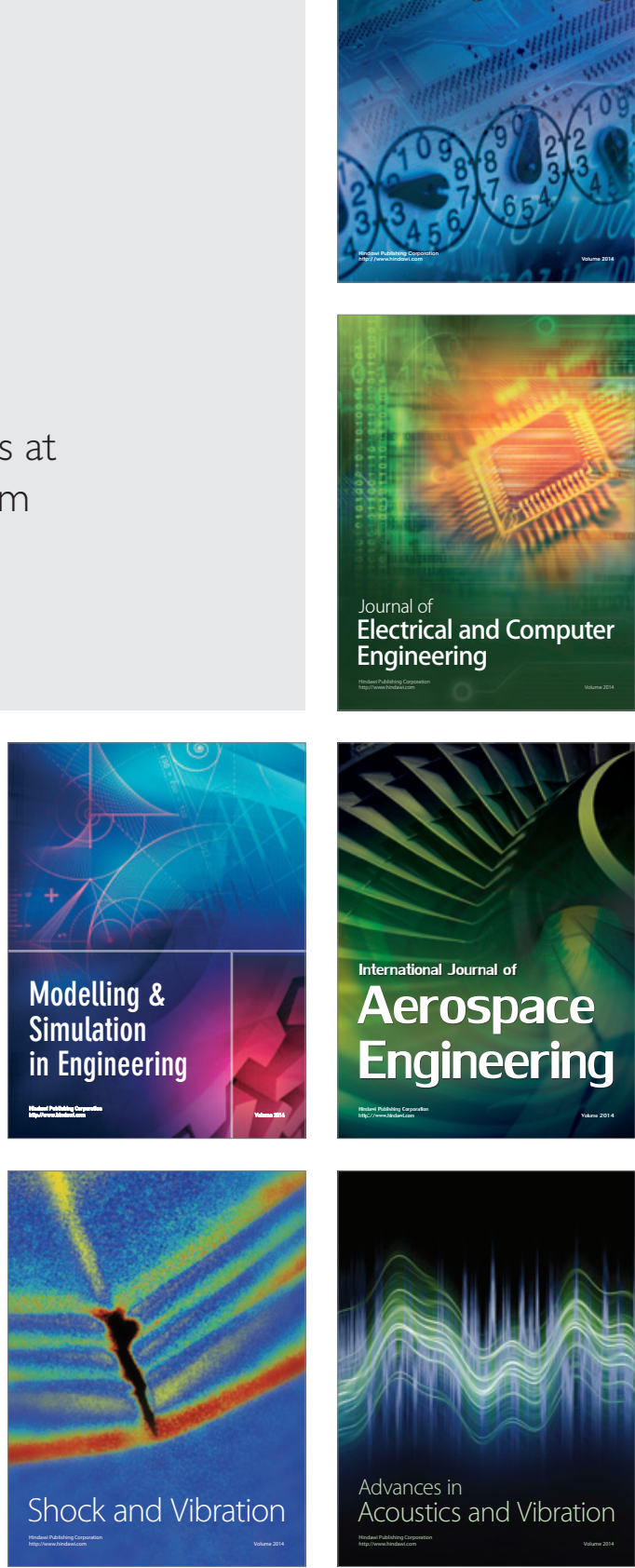